\title{
CARACTERIZAÇÃO DE GESTANTES EM ATENDIMENTO PRÉ-
} NATAL

\author{
Delma Pinheiro dos Santos ALVES ${ }^{1}$ \\ Gleidson Brandão OSELAME ${ }^{2}$ \\ Denecir de Almeida DUTRA ${ }^{3}$ \\ Alessandra Andréa da Silva TETZAFF ${ }^{4}$ \\ Elia Machado de OLIVEIRA ${ }^{5}$
}

\begin{abstract}
${ }^{1}$ Enfermeira. Centro Universitário Campos de Andrade. Curitiba - PR. Contato: delma_p_alves@ hotmail.com
${ }^{2}$ Enfermeiro. Docente pelo Centro Universitário Campos de Andrade. Curitiba - PR. Contato: gleidsonoselame@gmail.com

${ }^{3}$ Geógrafo. Docente pelo Centro Universitário Campos de Andrade. Curitiba - PR. Contato: denecir.dutra@ @erra.com.br ${ }^{4}$ Enfermeira. Docente pelo Centro Universitário Campos de Andrade. Curitiba - PR. Contato: ale.tetzlaff@gmail.com

${ }^{5}$ Enfermeira. Coordenadora do curso de Enfermagem. Centro Universitário Campos de Andrade. Curitiba - PR. Contato: elia561@ hotmail.com
\end{abstract}

\section{Recebido em: 14/05/2015 - Aprovado em: 14/11/2015 - Disponibilizado em: 18/12//2015}

\begin{abstract}
RESUMO
Introdução: O pré-natal é a assistência multidisciplinar a mulher durante o ciclo gravídico puerperal com finalidade de orientá-la sobre hábitos de vida, dieta, atividade física, higiene e vestimenta, bem como orientação psicológica. Objetivo: Caracterizar gestantes em atendimento pré-natal e os fatores associados à adesão no primeiro trimestre de gestação. Métodos: Estudo descritivo exploratório com abordagem qualiquantitativa. Para a coleta de dados foi utilizado um questionário composto por 30 questões. Foram inclusas no estudo 30 gestantes em acompanhamento prénatal no primeiro trimestre de gestação. Resultados: Com relação à idade das gestantes, variou entre 15 e 20 anos $(n=12 ; 40 \%)$. Quanto ao estado civil, a união estável foi à predominante $(n=14 ; 46,7 \%)$. Observou-se que 43,3\% $(n=13)$ das gestantes eram usuárias de tabaco, álcool e drogas ilícitas como maconha, crack e cocaína. Das que apresentaram problemas gestacionais anteriores $(\mathrm{n}=10 ; 33,3 \%)$ destes foram doença hipertensiva específica da gravidez (DHEG). Em 23,3\% ( $\mathrm{n=07)}$ apresentaram problemas de saúde na gestação atual, com DHEG, pré-eclâmpsia, bronquite asmática e infecção urinária. Sobre o conhecimento quanto às doenças sexualmente transmissíveis, $46,7 \%(n=14)$ não sabem o que as complicações das doenças sexualmente transmissíveis trazem as gestantes e ao recém nascido. Relativo à adesão ao pré-natal 40,0\% (n=12) procurou a Unidade Básica de Saúde para vinculação ao programa por ser mais próximo de sua residência e $60 \%$ por ser totalmente gratuito. Ainda, $86,7 \%(\mathrm{n}=26)$ declaram satisfação com o atendimento. Conclusão: Conhecer o perfil destas gestantes é fator essencial para o sucesso da assistência.
\end{abstract}

Palavras-chave: Cuidado pré-natal. Gestantes. Enfermagem obstétrica. Saúde da mulher. Saúde pública.

\begin{abstract}
Introduction: Prenatal care is the multidisciplinary care to women during pregnancy to childbirth order to guide it on lifestyle, diet, physical activity, hygiene and clothing and counselling. Objective: To characterize pregnant women in prenatal care and factors associated with adherence in the first trimester of pregnancy. Methods: A descriptive exploratory study with quali-quantitative approach. For data collection was used a questionnaire composed of 30 questions. They were included in the study 30 pregnant women in prenatal care in the first trimester of pregnancy. Results: Regarding the age of the women ranged between 15 and 20 years $(\mathrm{n}=12 ; 40 \%)$. Regarding marital status, the stable union was the predominant $(n=14 ; 46.7 \%)$. It was observed that $43.3 \%(n=13)$ of pregnant women were tobacco users, alcohol and illicit drugs such as marijuana, crack and cocaine. Of those who had previous gestational problems $(n=10 ; 33.3 \%)$ of these were hypertensive disorders of pregnancy (HDP). In $23.3 \%(n=07)$ had health problems during the pregnancy with preeclampsia, asthmatic bronchitis and urinary tract infection. About knowledge about sexually transmitted diseases, $46.7 \%(\mathrm{n}=14)$ do not know what the complications of sexually transmitted diseases bring pregnant women and newborn. Concerning the accession to prenatal $40.0 \%(\mathrm{n}=12)$ sought the primary care unit for linking the program to be closer to his residence and $60 \%$ for being completely free. Still, $86.7 \%(\mathrm{n}=26)$ declare satisfaction with care. Conclusion: Knowing the profile of these pregnant women is an essential factor for the success of assistance.
\end{abstract}

Keywords: Prenatal care. Pregnant women. Midwifery. Women's health. Public health. 


\section{INTRODUÇÃO}

No Brasil, a qualidade da atenção obstétrica continua a ser um ponto crítico da assistência à saúde da mulher. Observa-se aumento da cobertura da assistência pré-natal e do número de consultas por gestantes nos últimos 15 anos, em que a proporção de gestantes sem acesso a qualquer consulta de pré-natal foi inferior a $2 \%$ no ano 2009 (MALTA et al., 2010).

Desta forma, um estudo avaliou os óbitos em menores de um ano no Brasil no período 1997-2006, utilizando a lista de mortes evitáveis por intervenções do Sistema único de Saúde (SUS), encontrou redução de todas as mortes evitáveis, exceto daquelas relacionadas a uma adequada atenção prénatal, sugerindo baixa qualidade dessa assistência (MALTA et al., 2010; DOMINGUES et al., 2012).

Neste sentido, a ausência de assistência pré-natal está associada à maior taxa de mortalidade perinatal. Cerca de $98 \%$ das mortes de mulheres por causas maternas são evitáveis pela adoção de medidas como a melhoria da qualidade no cuidado e a garantia do acesso ao serviço de saúde (GAIOSO et al., 2014).

Assim, o Ministério da Saúde destaca a importância desse acompanhamento e incentivam todas as gestantes a buscarem o atendimento gratuito no Sistema Único de
Saúde (SUS), sendo a Unidade Básica de Saúde (UBS) a porta de entrada preferencial da gestante, ponto de atenção estratégico para melhor acolher suas necessidades, proporcionando um acompanhamento longitudinal e continuado (GAIOSO et al., 2014).

O pré-natal é a assistência multidisciplinar a mulher durante o ciclo gravídico puerperal com finalidade de orientála sobre hábitos de vida, dieta, atividade física, higiene e vestimenta, bem como orientação psicológica, além de preparação para o parto, cuidados com o recém-nascido e amamentação. Inclui ainda realização de tratamento e diagnóstico de doenças preexistentes que podem se complicar ou se agravar no período gestacional (PASCHOAL, MANTOVANI e MÉIER, 2007).

Portanto, o presente estudo teve como objetivo caracterizar gestantes em atendimento pré-natal e os fatores associados à adesão no primeiro trimestre de gestação.

\section{METODOLOGIA}

Tratou-se de um estudo descritivo exploratório com abordagem qualiquantitativa. $\mathrm{O}$ campo selecionado para a realização da pesquisa foi uma Unidade Básica de Saúde (UBS) na cidade de CuritibaPR. 
Para a coleta de dados foi utilizado um questionário composto por 30 questões. Foram inclusas no estudo 30 gestantes em acompanhamento pré-natal na referida UBS em acompanhamento regular e no primeiro trimestre de gestação.

Foram seguidos os princípios éticos de pesquisa que envolve Seres Humanos, conforme preconiza a resolução 466/2012 do Conselho Nacional de Saúde. A pesquisa foi aprovada pelo Comitê de Ética em Pesquisa do Centro Universitário Campos de Andrade, sob o parecer consubstanciado número 0560 de 10/09/2014.

\section{RESULTADOS}

\section{Caracterização das gestantes}

Com relação à idade das gestantes, a maioria variou entre 15 e 20 anos $(n=12$; 40\%). Quanto ao estado civil, a união estável foi à predominante $(\mathrm{n}=14 ; 46,7 \%)$. As demais variáveis podem ser observadas na Tabela 1.

Tabela 1. Caracterização das gestantes em atendimento pré-natal. Curitiba, Paraná, Brasil 2015

\begin{tabular}{clcc}
\hline Variáveis & & n & \% \\
\hline \multirow{3}{*}{ Idade (anos) } & $>15$ & 02 & 6,7 \\
& $15-20$ & 12 & 40,0 \\
& $21-25$ & 08 & 26,7 \\
& $26-30$ & 07 & 23,3 \\
Raça & $36-40$ & 01 & 3,3 \\
\hline \multirow{2}{*}{ Estado civil } & Branca & 11 & 36,7 \\
& Parda & 10 & 33,3 \\
& Negra & 06 & 20,0 \\
& Amarela & 03 & 10,0 \\
\hline \multirow{3}{*}{ Número de filhos } & Casada & 04 & 13,3 \\
& União Estável & 14 & 46,7 \\
& Solteira & 12 & 40,0 \\
\hline & 01 & 12 & 40,0 \\
& 02 & 11 & 36,7 \\
& 03 & 05 & 16,7 \\
& 04 & 01 & 3,3 \\
& 05 & 01 & 3,3 \\
\hline \multirow{2}{*}{ Escolaridade } & Ensino & 12 & 40,0 \\
& Fundamental & 16 & 53,3 \\
& Ensino Médio & 02 & 6,7 \\
& Ensino Superior & & \\
\hline \multirow{2}{*}{ Incompleto } & &
\end{tabular}

\section{Aspectos relacionados a complicações gestacionais}

Observou-se que $43,3 \% \quad(n=13)$ das gestantes eram usuárias de tabaco, álcool e drogas ilícitas como maconha, crack e cocaína. Das que apresentaram problemas gestacionais anteriores $(\mathrm{n}=10 ; 33,3 \%)$ estes foram doença hipertensiva específica da gravidez (DHEG) parto prematuro, gestação 
ectópica, aborto espontâneo, infecção urinária, perda de líquido amniótico, ameaça de aborto e aborto.

Em 23,3\% $\quad(n=07)$ das gestantes possuem problemas de saúde na gestação atual, com DHEG, pré-eclâmpsia, bronquite asmática e infecção urinária. A Figura 1 apresenta a distribuição destas complicações.

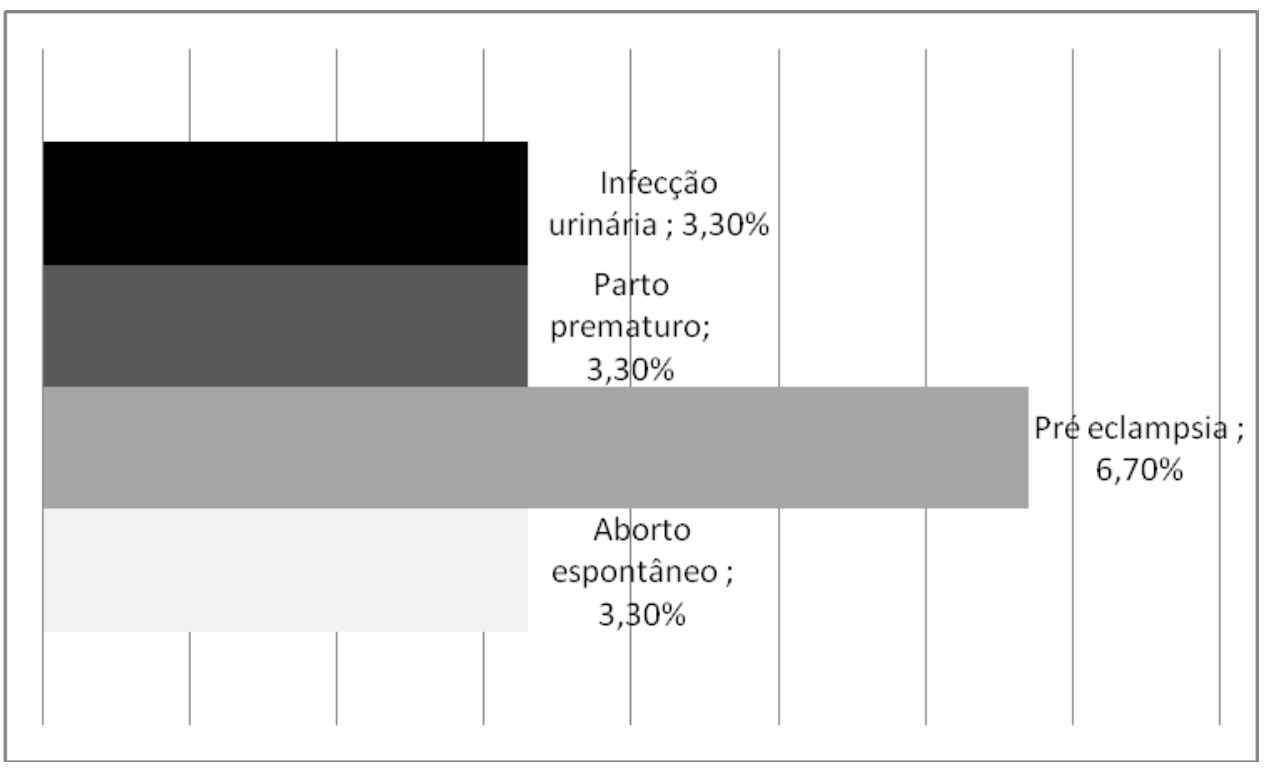

Figura 1. Complicações recorrentes apresentadas pelas gestantes. Curitiba, Paraná, Brasil, 2015.

Sobre o conhecimento quanto às doenças sexualmente transmissíveis, $46,7 \%$ $(\mathrm{n}=14)$ responderam que não sabem o que as complicações das doenças sexualmente transmissíveis trazem as gestantes e ao recém nascido.

\section{Adesão ao pré-natal}

Relativo à adesão ao pré-natal 40,0\% $(\mathrm{n}=12)$ procurou a Unidade Básica de Saúde para vinculação ao programa por ser mais próximo de sua residência e $60 \%$ por ser totalmente gratuito por dispensar um atendimento humanizado.

Quando questionadas sobre as vantagens em realizar o pré-natal pelo SUS, $83,3 \%(\mathrm{n}=25)$ acreditam que existe vantagem, conforme os relatos:

[...] Sou pobre e o atendimento é perto da minha casa ali tive meus pré-natais, quem não pode comprar ganha o enxoval do bebê. (Azul Marinho).

[...] Porque não tenho condições financeiras para pagar um plano. (Azul piscina). 
[...] Porque para quem não tem condições de pagar é tudo de bom. Eu que sou pobre fui bem atendida e encaminhada para os exames. (Rosa bordo).

[...] É fácil acesso e perto de casa, tudo gratuito com chance de ter conhecimento. (Mesclado).

Uma parcela das gestantes $(n=5$; $16,7 \%$ ) manifestou existir desvantagens conforme os relatos:

[...] Não existe vantagem, pois no particular você pode optar por cesária ou parto normal, apartamento ou alojamento conjunto no SUS você é tratado como qualquer um se não pode pagar tem que esperar a vontade dos médicos. (Verde oliva).

[...] Os exames são demorados, as consultas cansativas, as enfermeiras mal humoradas, tratam a gente igual um lixo. (Vermelho).

[...] A fila de espera é muito grande e a falta de consideração da parte dos funcionários é bem visível. (Verde mate).

Não podemos deixar de destacar a expectativa e o que as gestantes entrevistadas esperam, em realizar o atendimento pelo SUS destacamos alguns relatos alguns relatos:

[...] Espero ser bem atendida no hospital, não sofrer e ter meu bebe saudável, espero fazer todos s exames ecografias e que dê tudo certo com o bebe e comigo, a saúde dele é muito importante pra mim. (Vermelho).

[...] Espero um atendimento humanizado pra mim e pro meu bebe que ele nasça com saúde. (Amarelo).

[...] Nenhuma!!! Expectativa Porque esperei uma consulta com cardiologista e mesmo com a carta da médica pedindo urgência não tive resultados, muitas mães perdem seus bebes até mesmo na hora do parto, porque os médicos ficam aguardando datas mesmo com as mães sentindo dores ou tendo situações de risco para os médicos isso é normal. Isso é um absurdo. (Amarelo ouro).

Quanto ao grau de satisfação com o atendimento pré-natal na rede mãe curitibana, $86,7 \%(\mathrm{n}=26)$ declaram satisfação com o atendimento, conforme os relatos:

[...] Apesar de tudo é $100 \%$ gratuito e perto de casa. (Vermelho).

[...] Atende a população carente. (Verde limão).

[...] Você acaba aprendendo com tudo é um conhecimento a mais. (Preto).

No entanto, $13,3 \% \quad(n=4)$ declaram insatisfação com as seguintes justificativas:

[...] Demora demais para sair os exames. (Amarelo). 
[...] Falta estrutura e humanização. (Rosa bebe).

[...] Porque se você precisa de algum exame tem que esperar muito na fila de espera. (Bege).

\section{DISCUSSÃO}

A idade predominante das gestantes estudadas variou entre 15 a 20 anos de idade. Destaca-se que atualmente, os índices de atendimento do Sistema Único de Saúde (SUS) demonstram o crescimento do número de internações para atendimento obstétrico nas faixas etárias de 10 a 14,15 a 19 e 20 a 24 anos (HENN e PICCININI, 2013).

Relativo à escolaridade das gestantes, $53,3 \%$ possuíam ensino médio e $40 \%$ o ensino fundamental. Ressalta-se que a maternidade precoce pode comprometer o processo de escolarização principalmente em condições socioeconômicas desfavoráveis, sobretudo na faixa etária predominante no estudo (ROSSETTO, SCHERMANN e BÉRIA, 2014).

Os estudos demográficos demonstram que há maiores riscos de complicações à saúde da mulher nas populações mais pobres e com um menor nível de educação, o que diminui a qualidade de vida da família. Os vários significados atribuídos para a qualidade de vida estão de acordo com as perspectivas econômicas, demográficas, antropológicas, bioética e, mais recentemente, ambiental e de saúde pública (NARCHI, 2010).

O uso de drogas foi citado por $43,3 \%$ das gestantes, fato que pode ser considerado alto quando relacionado ao risco que esta ação proporciona ao feto. Tal fato vai de encontro com o crescimento mundial no uso de drogas por gestantes, porém ainda é um problema de saúde pública pouco discutida. O uso de drogas na gestação pode ser mais devastador, visto que resulta em consequências irreversíveis em alguns casos ao binômio mãe-bebê (YAMAGUCHI et al., 2008).

Desde a década de oitenta, os especialistas têm apontado vários fatores que estariam influenciando o uso de álcool e drogas e destacam os socioeconômicos e políticos, como o desemprego, insegurança do indivíduo em relação ao futuro, carência de alternativas de lazer e trabalho para jovens, a repressão política, a atividade educativa opressiva, ambas restringindo a postura crítica e a ação criativa. Tudo isso surge como fatores geradores de angústia e depressão, conduzindo à necessidade de evasão psicológica e a busca de satisfação propiciada por substancias como álcool e outras drogas psicoativas (RODRIGUES, NASCIMENTO e ARAÚJO, 2011).

Na mãe, sabe-se que as fumantes, por exemplo, têm menor produção de leite. Além disso, o uso do cigarro está associado com problemas de reprodução, podendo causar 
placenta prévia, deslocamento prematuro de placenta e ruptura prematura de membranas (RODRIGUES, NASCIMENTO e ARAÚJO, 2011).

Dentre as gestantes avaliadas, $23,7 \%$ apresentaram problemas gestacionais. As mortes maternas relacionadas às causas obstétricas são classificadas em dois tipos, as causas obstétricas e as obstétricas indiretas. As causas diretas são aquelas resultantes de complicações relacionadas ao ciclo gravídico puerperal, ocorrendo devido a tratamento incorreto, omissão, intervenção, ou resultantes de uma série de eventos. As mortes por causas obstétricas indiretas são resultantes de doenças que existiam antes da gestação ou que se desenvolveram durante esta e que são agravadas pelas alterações fisiológicas próprias da gestação (GAIOSO et al., 2014).

As patologias que mais acometem as gestantes geralmente produzem manifestações clinicamente detectáveis no decurso da gestação. Habitualmente os sinais e sintomas aparecem apenas no último trimestre da gravidez, quando as alterações patológicas encontram-se num estágio avançado, determinando condições ameaçadoras à vida da mãe e/ou do concepto, expondo as gestantes desprovidas de assistência especializada a situações de urgências/emergências obstétricas, exigindo intervenções imediatas e em alguns casos até mesmo a interrupção da gravidez (SMELTZER e BARE, 2005).

Sobre doenças sexualmente transmissíveis (DST) 46,7\% referiram não ter conhecimento sobre as causas e riscos envolvidos. As DST's apresentam prevalência significativa tanto na população geral quanto nas gestantes. Nestas devem-se considerar as alterações fisiológicas em seu organismo que podem, inclusive, alterar o curso das doenças. Complicações obstétricas e neonatais podem ocorrer em decorrência delas, acarretando aumento de morbimortalidade materno infantil (FONTE et al., 2012).

Foram notificados no Brasil em 2010, 5.666 casos de HIV em gestantes, com taxa de detecção de 2,0 casos por 1.000 nascidos vivos. Em 2010, a única região com uma taxa de detecção de HIV em gestantes superior à média nacional foi a Região Sul com 4,8 casos por 1.000 nascidos vivos (FONTE et al., 2012).

A adesão das mulheres ao pré-natal está relacionada com a qualidade da assistência prestada pelos serviços e profissionais de saúde. Por ocorrer em um corpo feminino em um contexto social impregnado por questões de gênero, fatores econômicos capazes de interferir no processo saúde-doença, a gravidez embora não seja considerada uma doença, pode levar ao adoecimento e morte. Conhecer o que as mulheres pensam sobre o pré- natal, 
(principalmente as que não aderem) assim como os aspectos biopsicossociais envolvidos é de fundamental importância para a qualidade de vida da gestante (VIEIRA et al., 2011).

Como recomendação do PAISM, os serviços já deveriam desenvolver discussão permanente com a população adstrita, especialmente com as mulheres, sobre a importância da assistência pré-natal, de forma a obter adesão das gestantes ao serviço de prénatal ainda no primeiro trimestre de gravidez (ROECKER et al., 2012).

É um modo diferenciado de cuidar da família permitindo maior proximidade $\mathrm{e}$ desenvolvimento de ações de promoção e prevenção sendo então uma excelente oportunidade de compreender e intervir no modo de vida do indivíduo e seus problemas sociais e emocionais (ROECKER et al., 2012).

Neste sentido a consulta de enfermagem, apresenta-se como instrumento de suma importância, por ter o objetivo de garantir a extensão da cobertura e melhoria da qualidade pré-natal, principalmente por meio da introdução das ações preventivas e promocionais às gestantes. É solicitado do profissional além de competência técnico cientifica e sensibilidade para compreender o ser humano e seu modo de vida e habilidade de comunicação, baseada na escuta e ação dialógica (SHIMIZU e LIMA, 2009).

A consulta de enfermagem proporciona a orientação de medidas favoráveis que visam à abordagem apropriada das necessidades peculiares das mulheres com quem os profissionais interagem em consultas no pré-natal, nas unidades básicas de saúde (DE ANDRADE BARBOSA, GOMES e DIAS, 2011).

Torna-se importante reforçar a importância da educação em saúde quando para as mulheres, já que a consulta pré-natal é um momento de reciprocidade educativa.

\section{CONCLUSÃO}

Identificaram-se fatores que mostram a falta de conhecimento das gestantes e sua vulnerabilidade durante a gestação.

A assistência do enfermeiro é um ponto importante da atenção básica, proporcionando acolhimento e atendimento longitudinal, visando evitar problemas ao longo do período gestacional ao puerpério.

Desta forma, conhecer o perfil destas gestantes é fator essencial para o sucesso da assistência. 


\section{REFERÊNCIAS}

DE ANDRADE BARBOSA, T. L.; GOMES, L. M. X.; DIAS, O. V. O pré-natal realizado pelo enfermeiro: a satisfação das gestantes. Cogitare Enfermagem, v. 16, n. 1, p. 29-35, 2011. ISSN 2176-9133.

DOMINGUES, R. M. S. M. et al. Avaliação da adequação da assistência pré-natal na rede SUS do Município do Rio de Janeiro, Brasil Adequacy of prenatal care in the National Health System in the city of Rio de Janeiro, Brazil. Cad. saúde pública, v. 28, n. 3, p. 425-437, 2012.

FONTE, V. R. F. et al. Conhecimento de gestantes de um hospital universitário relacionado à prevenção de DST/AIDS. Rev. enferm. UERJ, v. 20, n. 4, p. 493-499, 2012. ISSN 0104-3552.

GAIOSO, E. M. S. et al. Gestantes atendidas em hospital de referência do SUS: quem são e quais os motivos? Journal of Management and Primary Health Care, v. 5, n. 1, p. 33-9, 2014.

HENN, C. G.; PICCININI, C. A. Adolescência e função paterna: Da gestação ao primeiro ano de vida do bebê. Estudos de Psicologia, v. 18, n. 4, p. 579-588, 2013. ISSN 1413-294X.

MALTA, D. C. et al. Atualização da lista de causas de mortes evitáveis por intervenções do Sistema Único de Saúde do Brasil.

Epidemiologia e Serviços de Saúde, v. 19, n. 2, p. 173-176, 2010. ISSN 1679-4974.

NARCHI, N. Z. Atenção pré-natal por enfermeiros na Zona Leste da cidade de São Paulo, Brasil. Rev Esc Enferm USP, v. 44, n. 2, p. 266-73, 2010.

PASCHOAL, A. S.; MANTOVANI, M. F.; MÉIER, M. J. Percepção da educação permanente, continuada e em serviço para enfermeiros de um hospital de ensino. Rev Esc Enferm USP, v. 41, n. 3, p. 478-84, 2007.

RODRIGUES, E. M.; NASCIMENTO, R.; ARAÚJO, A. Protocolo na assistência pré-natal: ações, facilidades e dificuldades dos enfermeiros da Estratégia de Saúde da Família. Rev Esc

Enferm USP, v. 45, n. 5, p. 1041-7, 2011.

ROECKER, S. et al. Demandas assistenciais frente à gestação e o nascimento de bebês com malformação. Revista de Enfermagem da UFSM, v. 2, n. 2, p. 252-263, 2012. ISSN 21797692.

ROSSETTO, M. S.; SCHERMANN, L. B.; BÉRIA, J. U. Maternidade na adolescência: indicadores emocionais negativos e fatores associados em mães de 14 a 16 anos em Porto Alegre, RS, Brasil. Revista Ciência \& Saúde Coletiva, v. 19, n. 10, 2014. ISSN 1413-8123.

SHIMIZU, H. E.; LIMA, M. G. The dimensions of prenatal care embodied in nursing consultation. Revista Brasileira de Enfermagem, v. 62, n. 3, p. 387-392, 2009. ISSN 0034-7167.

SMELTZER, S. C.; BARE, B. B. Tratado de Enfermagem Médico-Cirúrgica. $1^{\circ}$ edição: Rio de Janeiro: Editora Guanabara Koogan 2005.

VIEIRA, S. M. et al. Percepção das puérperas sobre a assistência prestada pela equipe de saúde no pré-natal. Texto and Contexto Enfermagem, v. 20, n. Esp., p. 255, 2011. ISSN 0104-0707.

YAMAGUCHI, E. T. et al. Drogas de abuso e gravidez. Rev Psiquiatr Clín, v. 35, n. Supl 1, p. 44-7, 2008. 